Griess: Ueber eine neue Reihe organischer Säuren. 453

\title{
Ueber eine neue Reihe organischer Säuren;
}

\section{von \\ Peter Griess.}

Schon öfters habe ich eine basische Verbindung von der Zusammensetzung $\mathrm{C}_{10} \mathrm{H}_{12} \mathrm{~N}_{2} \mathrm{O}_{3}$ erwähnt, welche neben anderen Producten entsteht, wenn man eine kalt gesättigte alkoholische Lösung von Amidobenzoësäure mit Cyangas behandelt. Von der Ansicht ausgehend, dass diese Verbindung durch Vereinigung von Cyansäure mit Amidobenzoësäure-Aethyläther entstanden sei, habe ich schon vor längerer Zeit versucht'), dieselbe durch Einwirkung von cyansaurem Kali auf salzsauren Amidobenzoësäure-Aethyläther darzustellen, nach folgender Gleichung:

$$
\underset{\begin{array}{c}
\text { Salzsaurer. Amido- } \\
\text { benzoesaure-Aethyläther. }
\end{array}}{\mathrm{C}_{9} \mathrm{H}_{11} \mathrm{NO}_{2}, \mathrm{HCl}}+\underbrace{\mathrm{Ka}, \mathrm{CNO}}_{\text {Kansaures }}=\underbrace{\mathrm{C}_{10} \mathrm{H}_{12} \mathrm{~N}_{2} \mathrm{O}_{3}}_{\begin{array}{c}
\text { Basisehe } \\
\text { Verbindung. }
\end{array}}+\underbrace{\mathrm{KaCl}}_{\begin{array}{c}
\text { Chlor- } \\
\text { kalium. }
\end{array}}
$$

Obwohl sich nun diese Reaction auch ganz genau im Sinne der gegebenen Gleichung vollzog, so zeigte sich doch, dass der dabei entstehende Körper $\mathrm{C}_{10} \mathrm{H}_{12} \mathrm{~N}_{2} \mathrm{O}_{3}$, was seine chemischen und physikalisehen Eigenschaften anbelangt, von der gleich zusammengesetzten basischen Ver indung durchaus verschieden ist. Ich habe bereits früher näher dargethan ${ }^{2}$ ), dass derselbe als Aethyläther der. Uramidobenzoësäure zu betrachten ist.

Nachdem ich so gefunden hatte, dass die Synthese der in Rede stehenden basischen Verbindung auf die angegebene Weise nicht ausgeführt werden kann, glaubte ich dieses Ziel durch Einwirkung von cyansaurem Aethyl auf Amidobenzoësäure erreichen zu können, gemäss nachstehender Gleichung:

$$
\underbrace{\mathrm{C}_{7} \mathrm{H}_{7} \mathrm{NO}_{2}}_{\begin{array}{c}
\text { Amidobenzoë- } \\
\text { saure. }
\end{array}}+\underbrace{\mathrm{C}_{3} \mathrm{H}_{5} \mathrm{NO}}_{\begin{array}{c}
\text { Cyansaures } \\
\text { Aethyl. }
\end{array}}=\underbrace{\mathrm{C}_{10} \mathrm{H}_{12} \mathrm{~N}_{8} \mathrm{O}_{3}}_{\begin{array}{c}
\text { Basische } \\
\text { Verbindung. }
\end{array}}
$$

1) Dies Journal [2] 4, 294.

2) A. a O. S. 293. 
454 Griess: Ueber eine nene Reihe organischer Säuren.

Der Versuch hat ergeben, dass sich diese beiden Körper in der That mit Leichtigkeit zu einander addiren, dass aber das so entstehende Product weder mit der basischen Verbindung, noch auch mit dem Uramidobenzoësäure-Aether identisch ist. Diese dritte Verbindung von der Zusammensetzung $\mathrm{C}_{10} \mathrm{H}_{12} \mathrm{~N}_{2} \mathrm{O}_{3}$ zeigt die Eigenschaften einer Säure, welche sich mit Basen zu wohJ charakterisirten Salzen vereinigt. In dem Nachfolgenden soll dieselbe etwas näher beschrieben werden.

Die Darstellung dieser neuen Säure ist äusserst einfach und geschieht am besten in der Weise, dass man Amidobenzoësäure in kalt gesättigter alkoholischer Lösung mit cyansaurem Aethyl - beide Körper im Verhältniss ihrer Atomgewichte genommen -, versetzt. Es tritt dabei Wärmeentwicklung ein und nach Verlauf von ungefähr 12 Stunden erhält man eine reichliche Ausscheidung weisser Krystalle, welche die neue Säure in einem fast reinen Zustande darstellen. Trennt man diese Krystalle von der Mutterlauge und lässt man letztere dann verdunsten, so wird eine weitere Quantität der Säure erhalten. Einmaliges Umkrystallisiren derselben ans sehr verdünntem Weingeist, wenn nöthig unter Zusatz von etwas Thierkohle, liefert sie sofort rein zur Analyse. Die für dieselbe aufgestellte Formel $\mathrm{C}_{10} \mathrm{H}_{12} \mathrm{~N}_{2} \mathrm{O}_{3}$ wird durch die nachstehenden analytischen Resultate bestätigt:

0,356 Grm. bei $110^{0}$ getrocknot gaben 0,753 Grm. Kohlensäure und 0,1855 Grm. Wasser. 0,312 Grm. Gaben 33,5 Ce. Stickstoff von $0^{\circ}$ und $760 \mathrm{Mm}$. Druek.

\begin{tabular}{lrcc} 
& & Berechnet & Gefunden \\
$\mathbf{C}_{10}$ & 120 & 57,69 & 57,68 \\
$\mathbf{H}_{18}$ & 12 & 5,77 & 5,79 \\
$\mathbf{N}_{2}$ & 28 & 13,46 & 13,49 \\
$\mathbf{O}_{3}$ & 48 & 23,08 & - \\
\cline { 2 - 4 } & 208 & $100,00$. &
\end{tabular}

Diese neue Säure ist selbst in kochendem Wasser nur sehr schwer löslich and krystallisirt daraus, als anch aus kochendem verdünnter. Weingeist in feinen weissen glänzenden Nadeln. Von heissem Alkohol wird sie sehr 
Griess: Ueber eine neue Reihe organischer Säuren. 455 leicht aufgenommen, von kaltem aber und Aether ziemlich schwierig. Lässt man ihre kalte alkoholische Lösung freiwillig an der Luft eindampfen, so erhält man die Säure in sechsseitigen Tüfelchen oder kleinen Prismen krystallisirt. Sie zeigt oine stark saure Reaction auf Pflanzenfarben und neutralisirt selbst die stärksten Basen vollständig. In höherer Temperatur schmilzt sie zu einem gelblichen Oele, welches sich bei längerem Erhitzen, unter Aufsehätumen und Entwicklung eines stechenden Geruchs, in eine neutrale, in allen gewöhnlichen Lösungsmitteln unlösliche Substanz verwandelt. Ausser dass sie davon gelöst wird, bewirkt selbst kochende concentrirte Salzsäure keine Veränderung dieser neuen Säure; sehr concentrirte Kalilauge dagegen zersetzt sie bei längerem Erhitzen unter Bildung ammoniakalisch riechender Dämpfe. Durch sehr starke Salpetersäure wird sie in eine in gelben Nadeln krystallisirende Nitroverbindung übergeführt.

Von den Salzen dieser Säure habe ich nar zwei dargestellt.

Bariumsalz $\left(\mathrm{C}_{10} \mathrm{H}_{42} \mathrm{~N}_{2} \mathrm{O}_{3}\right)_{2}$, Ba. Es krystallisirt in mikroskopischen Nädelchen, welche in der Regel warzenförmig vereinigt und selbst in kaltem Wasser leicht löslich sind.

$0,319 \mathrm{Grm}$. bei $110^{\circ}$ getrocknet gaben $0,1145 \mathrm{Grm}$. kohlensaures Barium.

$$
\begin{gathered}
\begin{array}{c}
\text { Berechnet } \\
\left(\mathrm{C}_{10} \mathrm{H}_{11} \mathrm{~N}_{2} \mathrm{O}_{3}\right)_{2}, \mathrm{Ba}
\end{array} \\
\mathrm{Ba}=24,86
\end{gathered}
$$

Im lufttrockenem Zustande enthält dieses Salz drei Mol. Krystallwasser.

$$
\underbrace{\left(\mathrm{C}_{10} \mathrm{HI}_{11} \mathrm{~N}_{2} \mathrm{O}_{3}\right)_{2}, \mathrm{Ba}+3 \mathrm{H}_{2} \mathrm{O}}_{3 \mathrm{H}_{2} \mathrm{O}=8,93} \quad \underset{9,25}{\text { Gefunden }}
$$

Silbersalz $\left(\mathrm{C}_{10} \mathrm{H}_{11} \mathrm{~N}_{2} \mathrm{O}_{3}\right)$, Ag. Versetzt man die sehr verdünnte heisse ammoniakalische Lösung der Säure mit salpetersaurem Silber, so scheidet sich dasselbe beim Erkalten in weissen schmalen Blättchen ab. 
456 Griess: Ueber eine neue Reihe organischer Sauren

$0,192 \mathrm{Grm}$. bei $110^{\circ}$ getrocknet hinterliesen beim Glähen 0,066 Grm. Ag.

$$
\underbrace{\left(\mathrm{C}_{10} \mathrm{H}_{11} \mathrm{~N}_{2} \mathrm{O}_{3}\right), \mathrm{Ag}}_{\mathrm{Ag}=34,37} \quad \begin{gathered}
\text { Grefunden } \\
34,29
\end{gathered}
$$

Bezüglich der Constitation dieser Sauure bemerke ich nur, dass ich geneigt bin, sie als Aethyluramidobenzoësäure aufzufassen, eine Ansicht, welche in der Formel $\left.\mathrm{C}_{6} \mathrm{H}_{4}\left(\begin{array}{c}\mathrm{CO} \\ \mathrm{C}_{2} \mathrm{H}_{5} \\ \mathrm{H}_{2}\end{array}\right\} \mathrm{N}_{2}\right)^{\prime},\left(\mathrm{CO}_{2} \mathrm{H}\right)^{\prime}$ ihren Ausdruck findet; wogegen ich den ihr isomeren Aether der Uramidobenzoësäure in folgender Weise constituirt betrachte:

$$
\left.\mathrm{C}_{10} \mathrm{H}_{12} \mathrm{~N}_{2} \mathrm{O}_{3}=\mathrm{C}_{6} \mathrm{H}_{4}\left(\begin{array}{c}
\mathrm{CO} \\
\mathrm{H}_{3}
\end{array}\right)_{\mathrm{N}_{2}}\right)^{\prime},\left(\mathrm{CO}_{2}, \mathrm{C}_{2} \mathrm{H}_{5}\right)^{\prime} \text {. }
$$

Was übrigens die rationelle Zusammensetzung der damit gleichfalls isomeren basischen Verbindung anlangt, so kann ich es bis jetzt nicht wagen, darüber auch nur eine Vermuthung auszusprechen.

Lässt man cyansaures Aethyl auf Amidodracylsäure und Anthranilsüure einwirken, so werden der vorerwähnten entsprechende Säuren erhalten, die ich jedoch vorläufig nicht näher besehreiben will. Ich bemerke nur, dass namentlich die so aus Amidodraçlsäure entstehende Säure der Aethyluramidobenzoessäure zum Verwechseln ahnlich ist.

\section{Nachsehrift.}

Cahours und Gal haben schon vor einiger Zeit eine Mittheilung „Ueber ejnige neue Körper; welche durch die Vereinigung von Cyansäure und rerschiedenen cyznsauren Aethern mit den Aethern der Amidosäuren der aromatischen Reihe entstehen', veröffentlicht. Die Resultate dieser Untersuchung waren mir bisher nur aus. zugsweise durch die Zeitschr. der Chem. 7, 282 bekannt geworden und erst vor einigen Tagen hatte ich Gelegenheit, 
die betreffende Originalabhandiung (Compt. rend. 71, 462) zu Gesicht zu bekommen. A us einer kurzen Bemerkung in der letztern schliesse iah nun, dass die genannten Chemiker die oben beschriebene Aethyluramidobenzoësäure ebenfalls bereits beobachtet haben müssen, indem sie sagen, dass sich auch „die freie Amidobenzoësäure, Amidocuminsäure und Amidoanissäure mit cyansaurem Aethyl verbinden können und dass die dabei entstehenden Körper denjenigen aus den betreffenden Aethern sehr ähnlich seien." Trotz dieser Bemerkung aber habe ich mich nicht entschliessen können, die Veröffentlichung der vor. liegenden Abhandlung zu unterdrücken und zwar deshalb nicht, weil jeh die darin beschriebenen Versuche ausführte, ohne von der betreffenden Angabe von Cahours und $\mathrm{Ga}$ l die geringste Kenntniss zu haben, ja zum Theil sehon lange vorher, ehe die gedachte Originalabhandlung dieser Chemiker im Druck ersohien.

Wie man leicht einsehen wird, stehen ja diese an und für sich sehr nahe liegenden Versuche im innigen $\mathrm{Zu}$ sammenhang mit den Arbeiten, welche mich sohon seit den letzten fünf Jahren beschäftigen, und deren Resultate ich zum Theil bereits in vorläufigen Notizen bekannt gemacht habe.

\section{Deber einen Aldehyd-Alkohol;}

\section{von \\ Ad. Wurtz.}

(Aus Compt. rend. 74, 1361; 1872.)

Cegenstand dieser Mittheilung ist ein Körper, welcher wenigstens unter den Substanzen, deren Constitution aufgeklărl ist, einem neuen Typus in der organischen Chemie anzugehören scheint. Derselbe ist ein Derivat des Aldehyds und spielt zugleich die Rolle eines Aldehyds and 